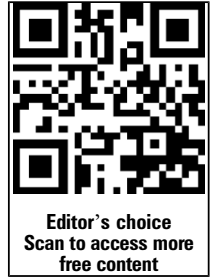

Institute of Environmental Medicine, Karolinska Institutet, Stockholm, Sweden

\section{Correspondence to} Dr Susanna C Larsson, Unit of Nutritional Epidemiology, Institute of Environmental Medicine, Karolinska Institutet, Box 210, Stockholm 17177 Sweden;

Susanna.Larsson@ki.se

Received 20 January 2015 Revised 12 July 2015

Accepted 19 July 2015 Published Online First

2 November 2015

\section{CrossMark}

To cite: Rahman I, Wolk A, Larsson SC. Heart

2015;101:1961-1965.

\title{
The relationship between sweetened beverage consumption and risk of heart failure in men
}

\author{
Iffat Rahman, Alicja Wolk, Susanna C Larsson
}

ABSTRACT

Objectives To investigate whether sweetened beverage consumption is associated with risk of heart failure (HF) in a large prospective population-based study of men. Methods and results A population-based cohort comprising 42400 men, 45-79 years of age, was followed from 1998 through 2010. Sweetened beverage consumption was assessed by utilising a food frequency questionnaire. Incident events of HF were identified through linkage to the Swedish National Patient Register and the Cause of Death Register. Cox regression analyses were implemented to investigate the association between sweetened beverage consumption and HF. During a mean follow-up time of 11.7 years, a total of 4113 HF events were identified. We observed a positive association between sweetened beverage consumption and risk of $\mathrm{HF}$ after adjustment for other risk factors ( $p$ for trend $<0.001$ ). Men who consumed two or more servings of sweetened beverages per day had a statistically significant higher risk of developing $\mathrm{HF}$ (23\%, 95\% Cl 1.12 to 1.35$)$ compared to men who were non-consumers.

Conclusions Our finding that sweetened beverage consumption is associated with higher risk of HF could have implications for HF prevention strategies. Additional prospective studies investigating the link between sweetened beverage consumption and HF are therefore needed.

\section{INTRODUCTION}

The prevalence of heart failure (HF) is estimated to be around 5.8 million in the USA, and more than 23 million people are affected worldwide. Moreover, the prevalence of HF has been rising, and is more prevalent among the elderly and males. ${ }^{1}$ Besides age and sex, cardiometabolic risk factors such as insulin concentrations, inflammatory markers, and blood pressure have been shown to be associated with increased HF risk. ${ }^{2-5}$

Sweetened beverage consumption has been shown to be associated with changes in blood pressure, concentrations of insulin, glucose, and C-reactive protein, and weight. ${ }^{6-12}$ Moreover, soft drink consumption has been associated with an increased risk of developing hypertension, metabolic syndrome, diabetes, coronary heart disease, and stroke. ${ }^{11}{ }^{13-17}$ Thus, high consumption of sweetened beverages may also increase the risk of HF. To the best of our knowledge, no prospective study has investigated the relationship between consumption of sweetened beverages and the risk of HF.
We therefore aimed to investigate whether sweetened beverage consumption is associated with HF risk in a large prospective population-based study of men.

\section{METHODS}

\section{Study population}

The study participants belonged to the Cohort of Swedish Men (COSM), a population-based cohort established between 1997 and 1998. All men born between 1918 and 1952 and residing in Örebro and Västmanland counties in Sweden were asked to complete an extensive questionnaire regarding physical activity, diet, anthropometric traits, and other lifestyle factors. In total, 48850 men answered the questionnaire.

We excluded those participants with incorrect or missing identification numbers $(\mathrm{N}=297)$, those who died before the start of follow-up $(\mathrm{N}=55)$, and those with implausible energy intakes (ie, 3 SDs from the $\log _{\mathrm{e}}$-transformed mean energy intake, $\mathrm{N}=535)$. We also excluded participants with prebaseline cancer (except non-melanoma skin cancer, $\mathrm{N}=2592$ ), HF or myocardial infarction (based on linkage of the cohort to the Swedish National Patient Register, $\mathrm{N}=2971$ ) because the diagnosis of these conditions may lead to changes in dietary pattern and beverage consumption. After theses exclusions, the final study population comprised 42400 men.

\section{Assessment of diet and beverage}

Diet and beverage consumption was assessed in 1997 with a food frequency questionnaire (FFQ), designed to assess the Swedish diet, in which participants reported their average consumption of 96 foods and beverage during the past year. For assessment of commonly consumed beverage, such as soft drinks and coffee, study participants were asked to report their daily or weekly intake of a standard $200 \mathrm{~mL}$ (one glass) serving. Sweetened beverage consumption was measured by asking the participants the following question: "How many soft drinks or sweetened juice drinks do you drink per day or per week"? Fruit juice was not included in our definition of sweetened beverages.

\section{HF ascertainment}

Dates of the first registered incident HF hospitalisation as well as dates of deaths from HF were ascertained from 1 January 1998 to 31 December 2010 through linkage of the cohort to the Swedish National Patient Register and the Cause of Death Register. The Swedish National Patient Register 
represents the inpatient register, which includes all hospital admissions that entailed at least one overnight stay, and the outpatient register which includes diagnoses registered during nonprivate specialised care. For identification of HF incidence we used International Classification of Diseases-10 codes I50 and I11.0. We included incident HF events recorded in the registers listed as either the primary or secondary diagnosis of hospitalisation or death. The study has been approved by the Regional Ethical Review Board at Karolinska Institutet, Sweden, and completion of the self-administered questionnaire was considered to imply informed consent.

\section{Assessment of covariates}

The questionnaire included information on participant's educational attainment, family history of myocardial infarction, diagnosis of hypertension, diagnosis of diabetes (which was complemented with information from the diabetes register and the Swedish National Patient Register), smoking and drinking habits, weight, height, physical activity, and diet. Body mass index (BMI) was calculated as the weight (in kilograms) divided by the square of height (in metres). Physical activity was assessed by reported time spent at each activity per day (eg, work, walking/bicycling, exercise, housework, sleeping) and was multiplied by its typical energy expenditure requirements. ${ }^{18}$ Information on diagnoses of stroke and angina was obtained from the Swedish National Patient Register.

\section{Statistical analyses}

Data handling and generation of descriptive statistics were performed in SAS (V.9.2; SAS Institute, Inc, Cary, North Carolina, USA). Descriptive statistics of the study population were presented as means and SDs for continuous variables, and $\mathrm{n}(\%)$ for categorical variables. Baseline characteristics were standardised to the age distribution of the total study population at baseline. Stata software V.12.1 (StataCorp) was used to calculate age-standardised rates and fit Kaplan-Meier survival methods and Cox proportional hazards regression. Kaplan-Meier survival methods were used to examine HF-free survival according to categories of sweetened beverage consumption. HRs and 95\% CIs were estimated using Cox proportional hazards regression models with attained age as the underlying time scale. All p values were two-sided, and a value of $p>0.05$ indicated the absence of a statistically significant effect. Follow-up was censored at the date of first event of HF, death or 31 December 2010, whichever occurred first. The proportional hazards assumption was examined by investigating Schoenfeld's residuals.

Sweetened beverage consumption was divided into five different categories. The categorisation for the four upper groups was based on the nearest whole or half value of quartiles of sweetened beverage consumption among those who consumed sweetened beverages. This was done in order to make the categorisations of servings per day more interpretable. The reference category was no consumption of sweetened beverage.

Covariates included in the multivariable-adjusted model were: educational attainment (primary school, high school, university), smoking (never, past, current $(\leq 10,>10$ cigarettes/day)), alcohol consumption (never/past/current $(<5, \geq 5 \mathrm{~g} /$ day)), total physical activity (MET (metabolic equivalent) in hours/day categorised into quartiles), family history of myocardial infarction, history of stroke, history of angina, hypertension, diabetes, BMI $\left(<18.5,18.5-24.9,25-29.9, \geq 30 \mathrm{~kg} / \mathrm{m}^{2}\right)$, coffee consumption (cups/day), fruit intake (servings/day), vegetable intake (servings/ day), processed meat consumption (g/day), fish consumption (servings/day), and total energy intake (kcal/day). The outcome variable was incident HF event. We carried out trend tests by assigning the median value for each category of sweetened beverage consumption and entered this variable as a continuous variable in the model. We also investigated if smoking status, being overweight, and age $>65$ years were potential effect modifiers in the association between sweetened beverage consumption and HF incidence by including them as interaction terms in the model.

To assess the potential effect of reverse causality - that is, undiagnosed HF contributes to subsequent changes in consumption of sweetened beverage-we conducted a sensitivity analysis where participants with HF events that were diagnosed during the first 5 years of follow-up were excluded from the model.

Three separate sensitivity analyses in which we excluded participants with either diabetes, history of stroke, or participants with history of angina from the model were also performed since these diseases could have strong confounding effects on the association between sweetened beverage consumption and HF. Diabetic patients are at increased risk of HF and they may have reduced their sweetened beverage consumption after their diagnosis. We also conducted an analysis where diabetes was not included as a covariate since diabetes could potentially have a mediator effect-that is, lie in the causal pathway between sweetened beverage consumption and HF incidence.

\section{RESULTS}

During a mean follow-up time of 11.7 years (494 772 personyears) a total of 4113 incident HF events (3604 first events HF hospitalisations and $509 \mathrm{HF}$ deaths) were identified. Baseline characteristics of the cohort according to different categories of sweetened beverage consumption are presented in table 1 . There was a strong negative trend between sweetened beverage consumption and educational level: $19 \%$ of the non-consumers were university educated while only $10 \%$ of men belonging to the highest consumption group ( $\geq 2$ servings/day) were university educated.

Kaplan-Meier survival curves showing the association between HF-free survival for the lowest and highest categories of sweetened beverage consumption is provided in figure 1 . The figure suggests an inverse association between HF-free survival and the highest category of sweetened beverage consumption ( $\geq 2$ servings/day).

The results from the Cox proportional hazards regression investigating the relationship between different levels of daily sweetened beverage consumption and the risk of HF are provided in table 2 . Compared with no consumption of sweetened beverages, daily consumption of $\geq 2$ servings was significantly associated with higher risk of HF (HR 1.23, 95\% CI 1.12 to 1.35). In the secondary analysis excluding men with diabetes, the results did not change appreciably (HR 1.21, 95\% CI 1.10 to 1.34). No significant statistical interaction could be found between any of the categories of sweetened beverage consumption and smoking, overweight, or age $>65$ years $(p>0.05)$. The HR for the interaction term between more than two cups of daily servings and smoking was 1.00 (95\% CI 0.74 to 1.36 ), 1.09 (95\% CI 0.92 to 1.31) for overweight, and 0.93 (95\% CI 0.82 to 1.05 ) for age $>65$ years.

The sensitivity analysis-where we excluded study participants who developed HF in the first 5 years of follow-up to account for potential reverse causality-showed results that were very similar to the main analysis. The HR for consumption of $\geq 2$ servings per day changed from 1.23 in the main analysis to 1.25 (95\% CI, 1.12 to 1.40 ) in the sensitivity analysis. 
Table 1 Age-standardised baseline characteristics of the study population of the Cohort of Swedish Men by categories of sweetened beverage consumption

\begin{tabular}{|c|c|c|c|c|c|}
\hline \multirow[b]{2}{*}{ Characteristic } & \multicolumn{4}{|c|}{ Sweetened beverage consumption (servings/day) } & \multirow[b]{2}{*}{$\geq 2(n=5546)$} \\
\hline & $0(n=21006)$ & 0 to $<0.5(n=7138)$ & 0.5 to $<1.0(n=3150)$ & 1.0 to $<2.0(n=5560)$ & \\
\hline Age at study entry* & $61(9.5)$ & $58(9.1)$ & $59(9.2)$ & $60(9.7)$ & $60(9.6)$ \\
\hline Ever smoker, \% & $13864(66)$ & $4283(60)$ & $1953(62)$ & $3336(60)$ & $3660(66)$ \\
\hline Obesity, \% & $9453(45)$ & $3283(46)$ & $1512(48)$ & $2613(47)$ & $2607(47)$ \\
\hline Diabetes, \% & $1470(7)$ & $214(3)$ & $126(4)$ & $278(5)$ & $332(6)$ \\
\hline Hypertension, \% & $4621(22)$ & $1499(21)$ & $756(24)$ & $1334(24)$ & $1387(25)$ \\
\hline University education, $\%$ & 3991 (19) & $1428(20)$ & $441(14)$ & $890(16)$ & $555(10)$ \\
\hline Total physical activity (MET, hours/day) & $41.4(4.8)$ & $41.1(4.6)$ & $41.9(4.8)$ & $41.9(5.0)$ & $42.6(5.5)$ \\
\hline Current drinkers, \% & $19746(94)$ & $6638(93)$ & $2898(92)$ & $4948(89)$ & $4880(88)$ \\
\hline History of stroke, \% & $420(2)$ & $143(2)$ & $63(2)$ & $111(2)$ & $166(3)$ \\
\hline History of angina, \% & $840(4)$ & $286(4)$ & $126(4)$ & $167(3)$ & $222(4)$ \\
\hline Family history of myocardial infarction before age $60, \%$ & $7772(37)$ & $2427(34)$ & $1134(36)$ & $1946(35)$ & $2107(38)$ \\
\hline Coffee consumption ( $\geq 3$ cups/day), \% & $13024(62)$ & $4140(58)$ & $1733(55)$ & $3225(58)$ & $3494(63)$ \\
\hline Fruit intake (servings/day) & $1.5(1.2)$ & $1.5(1.1)$ & $1.5(1.1)$ & $1.5(1.2)$ & $1.5(1.2)$ \\
\hline Vegetable intake (servings/day) & $2.7(1.8)$ & $2.7(1.6)$ & $2.6(1.6)$ & $2.6(1.7)$ & $2.4(1.7)$ \\
\hline Processed meat consumption (g/day) & $38.6(30.1)$ & $40.7(27.7)$ & $43.6(31.2)$ & $43.9(32.4)$ & $44.6(32.0)$ \\
\hline Fish consumption (servings/week) & $1.9(2.03)$ & $1.9(1.5)$ & $1.8(1.5)$ & $1.9(2.1)$ & $1.9(2.0)$ \\
\hline
\end{tabular}

\section{DISCUSSION}

In this large, prospective, population-based study of men we found sweetened beverage consumption to be significantly positively associated with the development of HF. Men who consumed at least two servings per day of sweetened beverages had a $23 \%$ higher risk of HF compared to non-consumers.

There are several potential biological mechanisms that could elucidate the observed association between sweetened beverage consumption and HF risk. Sweetened beverage consumption has been shown to be associated with several cardiometabolic risk factors, ${ }^{10}$ including blood pressure, ${ }^{7} 9$ concentrations of insulin, ${ }^{8}$ glucose, ${ }^{67}$ and C-reactive protein, ${ }^{6}$ and weight gain. ${ }^{12}$ All of these factors have been shown to be associated with the risk of $\mathrm{HF}^{2-5}$ Sweetened beverages have moderately high glycaemic load, ${ }^{19}$ glycaemic control has been suggested to decrease possibly the risk of $\mathrm{HF}^{20}$ and a positive association between

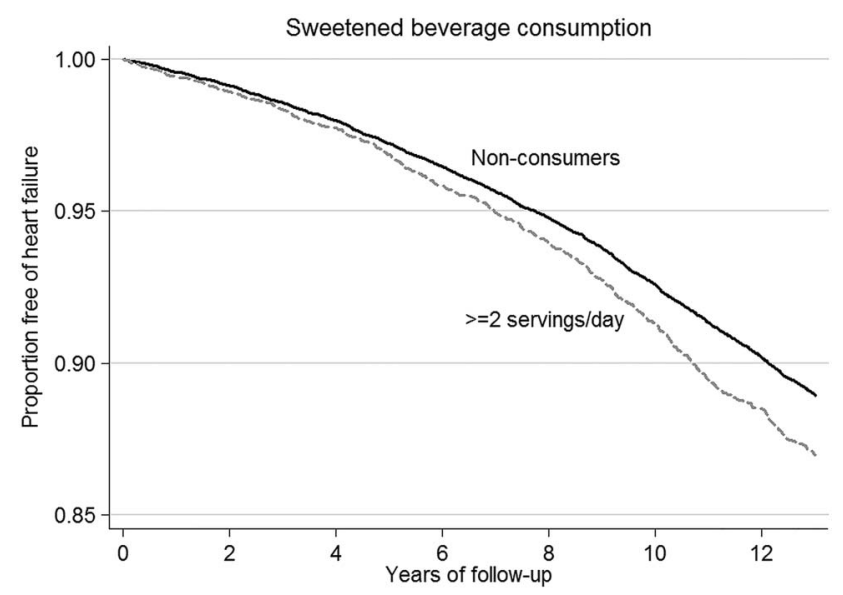

Figure 1 Heart failure-free survival for the highest and lowest categories of sweetened beverage consumption. glycaemic load and HF has previously been reported, although the result did not reach statistical significance. ${ }^{21}$

The strengths of our study include the large population-based cohort and the prospective design. The cohort had a long follow-up time and we could identify a large number of both incident HF hospitalisations and HF deaths. The definition of HF was based on clinical diagnoses and a validation study on primary HF diagnoses in the Swedish National Patient Register has shown that the validity for a primary HF diagnosis is high $(95 \%) .^{22}$

A potential limitation that applies to this study is that the assessment of sweetened beverage consumption was based on a self-administered FFQ. Therefore, there could have been some degree of misclassification of sweetened beverage consumption. However, due to the prospective design of the cohort we do not expect a potential misclassification to be dependent on the outcome. Thus, a potential misclassification of sweetened beverage consumption would most likely have attenuated the association with HF risk. It is also worth mentioning that a previous validation study has examined the correlations between the FFQ used in this study and the mean of 14 24-h recall interviews for different macronutrients. The study reported that the correlation coefficients for nutrient intake ranged from $0.44-0.81$, with a mean of $0.65 .^{23}$ In addition, the question on sweetened beverage consumption encompasses both sugar sweetened and artificially sweetened beverages; hence, we could not differentiate between the two in our study and could not make any inferences on whether any of the two particular sweetened beverage types were exclusively associated with HF risk.

It would also have been valuable to have biomarker measurements to gain a deeper understanding of the biological mechanisms behind the observed association. Biomarkers such as blood pressure, insulin, glucose, and CRP concentrations could potentially have mediated the effect of sweetened beverage consumption and HF. 
Table 2 Association between sweetened beverage consumption and risk of heart failure in men

\begin{tabular}{|c|c|c|c|c|c|c|}
\hline & \multicolumn{4}{|c|}{ Sweetened beverages consumption servings/day } & \multirow[b]{2}{*}{$\geq 2$} & \multirow[b]{2}{*}{$p$ for trend } \\
\hline & 0 & 0.1 to $<0.5$ & 0.5 to $<1.0$ & 1.0 to $<2.0$ & & \\
\hline \multicolumn{7}{|l|}{ Total population } \\
\hline Number of cases & 2123 & 490 & 271 & 572 & 657 & - \\
\hline Person-years & 242796 & 86234 & 37612 & 64757 & 63371 & - \\
\hline Incidence rate* & 921 & 813 & 984 & 985 & 1130 & - \\
\hline Age-adjusted HR (95\% Cl) & 1.00 (reference) & 0.88 (0.80 to 0.97$)$ & 1.03 (0.91 to 1.17$)$ & 1.07 (0.97 to 1.17$)$ & 1.26 (1.15 to 1.37$)$ & $<0.001$ \\
\hline Multivariable-adjustedt HR (95\% Cl) & 1.00 (reference) & 0.98 (0.88 to 1.08$)$ & 1.08 (0.95 to 1.23$)$ & 1.09 (0.99 to 1.20$)$ & 1.23 (1.12 to 1.35$)$ & $<0.001$ \\
\hline \multicolumn{7}{|l|}{ Non-diabetics } \\
\hline Number of cases & 1776 & 455 & 243 & 515 & 574 & - \\
\hline Multivariable-adjustedt HR (95\% Cl) & 1.00 (reference) & 0.98 (0.89 to 1.09 ) & 1.05 (0.92 to 1.21$)$ & $1.10(0.99$ to 1.21$)$ & 1.21 (1.10 to 1.34 ) & $<0.001$ \\
\hline
\end{tabular}

Moreover, because of the observational study design we cannot make a causal interpretation; the association between sweetened beverage consumption and HF risk could have been due to unmeasured confounders or residual confounding at baseline or during follow-up time. We investigated the association between sweetened beverage consumption and HF risk in a population comprising middle-aged and elderly white men. The sweetened beverage consumption in the COSM was about the same as in other European populations, but lower than in US populations. ${ }^{24}$ Thus, our study findings might not apply to certain countries, younger age groups or women.

\section{CONCLUSIONS}

Our study findings suggest that sweetened beverage consumption could contribute to HF development. These findings could have implications for HF prevention strategies. Further prospective studies examining this relationship are therefore necessitated. Moreover, possible biological mechanisms linking sweetened beverage consumption with $\mathrm{HF}$ risk need to be studied carefully.

\section{Key messages}

What is already known on this subject?

Previous observational studies have shown that sweetened beverage consumption is associated with increased risk of coronary heart disease and stroke. The relationship between sweetened beverage consumption and heart failure is unknown.

What might this study add?

This prospective population-based study followed 42400 men and showed that sweetened beverage consumption is associated with risk of heart failure. Men who consumed at least two servings per day of sweetened beverages had a $23 \%$ higher risk of heart failure compared to non-consumers

\section{How might this impact on clinical practice}

These investigations could aid in heart failure risk prevention strategies by, for example, improving diet recommendations.
Contributors IR, SL, AW participated in the study design and in writing the manuscript. AW participated in the data collection. IR analysed the data and wrote the manuscript. IR, SL, AW interpreted the data and critically reviewed the paper. All authors read and approved the final manuscript.

Funding This work was supported by the Swedish Research Council Committee for Medicine and the Swedish Research Council Committee for Infrastructure.

Competing interests None declared.

Ethics approval Regional Ethical Review Board at Karolinska Institute.

Patient consent Obtained.

Provenance and peer review Not commissioned; externally peer reviewed.

\section{REFERENCES}

1 Bui AL, Horwich TB, Fonarow GC. Epidemiology and risk profile of heart failure. Nat Rev Cardiol 2011;8:30-41.

2 Eisen $A$, Benderly $M$, Behar $S$, et al. Inflammation and future risk of symptomatic heart failure in patients with stable coronary artery disease. Am Heart J 2014; 167:707-14.

3 Loehr LR, Rosamond WD, Poole C, et al. The potentially modifiable burden of incident heart failure due to obesity: the Atherosclerosis Risk in Communities study. Am J Epidemiol 2010;172:781-9.

4 MacDonald MR, Petrie MC, Hawkins NM, et al. Diabetes, left ventricular systolic dysfunction, and chronic heart failure. Eur Heart J 2008;29:1224-40.

5 Roger VL. Epidemiology of heart failure. Circ Res 2013;113:646-59.

6 Aeberli I, Gerber PA, Hochuli M, et al. Low to moderate sugar-sweetened beverage consumption impairs glucose and lipid metabolism and promotes inflammation in healthy young men: a randomized controlled trial. Am J Clin Nutr 2011;94:479-85.

7 Duffey KJ, Gordon-Larsen P, Steffen LM, et al. Drinking caloric beverages increases the risk of adverse cardiometabolic outcomes in the Coronary Artery Risk Development in Young Adults (CARDIA) study. Am J Clin Nutr 2010;92:954-9.

8 Janssens JP, Shapira N, Debeuf $P$, et al. Effects of soft drink and table beer consumption on insulin response in normal teenagers and carbohydrate drink in youngsters. Eur J Cancer Prev 1999;8:289-95.

9 Malik AH, Akram Y, Shetty S, et al. Impact of sugar-sweetened beverages on blood pressure. Am J Cardiol 2014;113:1574-80.

10 Malik VS, Popkin BM, Bray GA, et al. Sugar-sweetened beverages, obesity, type 2 diabetes mellitus, and cardiovascular disease risk. Circulation 2010;121:1356-64.

11 Mozaffarian D, Hao T, Rimm EB, et al. Changes in diet and lifestyle and long-term weight gain in women and men. N Engl J Med 2011;364:2392-404.

12 Yang Q, Zhang Z, Gregg EW, et al. Added sugar intake and cardiovascular diseases mortality among US adults. JAMA Intern Med 2014;174:516-24.

13 de Koning L, Malik VS, Kellogg MD, et al. Sweetened beverage consumption, incident coronary heart disease, and biomarkers of risk in men. Circulation 2012; 125:1735-41.

14 Eshak ES, Iso $\mathrm{H}$, Kokubo $\mathrm{Y}$, et al. Soft drink intake in relation to incident ischemic heart disease, stroke, and stroke subtypes in Japanese men and women: the Japan Public Health Centre-based study cohort I. Am J Clin Nutr 2012;96:1390-7.

15 Larsson SC, Akesson A, Wolk A. Sweetened beverage consumption is associated with increased risk of stroke in women and men. J Nutr 2014;144:856-60. 
16 Malik VS, Popkin BM, Bray GA, et al. Sugar-sweetened beverages and risk of metabolic syndrome and type 2 diabetes: a meta-analysis. Diabetes Care 2010;33:2477-83.

17 Sayon-Orea C, Martinez-Gonzalez MA, Gea A, et al. Baseline consumption and changes in sugar-sweetened beverage consumption and the incidence of hypertension: The SUN project. Clinical Nutrition 2014. pii: S0261-5614(14)00291-X.

18 Norman A, Bellocco R, Bergstrom A, et al. Validity and reproducibility of self-reported total physical activity- differences by relative weight. Int I Obes Relat Metab Disord 2001;25:682-8.

19 Atkinson FS, Foster-Powell K, Brand-Miller JC. International tables of glycemic index and glycemic load values: 2008. Diabetes Care 2008;31:2281-3.

20 Iribarren C, Karter AJ, Go AS, et al. Glycemic control and heart failure among adult patients with diabetes. Circulation 2001;103:2668-73.
21 Levitan EB, Mittleman MA, Wolk A. Dietary glycemic index, dietary glycemic load, and incidence of heart failure events: a prospective study of middle-aged and elderly women. J Am Coll Nutr 2010;1:65-71.

22 Ingelsson E, Arnlov J, Sundstrom J, et al. The validity of a diagnosis of heart failure in a hospital discharge register. Eur I Heart Fail 2005; 7: 787-91.

23 Messerer M, Johansson SE, Wolk A. The validity of questionnaire-based micronutrient intake estimates is increased by including dietary supplement use in Swedish men. J Nutr 2004;134:1800-5.

24 Lee JE, Hunter DJ, Spiegelman D, et al. Intakes of coffee, tea, milk, soda and juice and renal cell cancer in a pooled analysis of 13 prospective studies. Int I Cancer 2007;121:2246-51. 